PROCEEDINGS OF THE

AMERICAN MATHEMATICAL SOCIETY

Volume 131, Number 1, Pages 117-127

S 0002-9939(02)06696-0

Article electronically published on August 19, 2002

\title{
RANDOMISED CIRCULAR MEANS OF FOURIER TRANSFORMS OF MEASURES
}

\author{
JONATHAN M. BENNETT AND ANA VARGAS
}

(Communicated by Andreas Seeger)

\begin{abstract}
We explore decay estimates for $L^{1}$ circular means of the Fourier transform of a measure on $\mathbb{R}^{2}$ in terms of its $\alpha$-dimensional energy. We find new upper bounds for the decay exponent. We also prove sharp estimates for a certain family of randomised versions of this problem.
\end{abstract}

\section{INTRODUCTION}

We begin with a definition. Let $0<\alpha \leq 2$. The $\alpha$-dimensional energy of a positive measure $\mu$ on $\mathbb{R}^{2}$ is defined by

$$
I_{\alpha}(\mu)=\int_{\mathbb{R}^{2}} \int_{\mathbb{R}^{2}} \frac{d \mu(x) d \mu(y)}{|x-y|^{\alpha}} .
$$

As in Wolff [13], we make the following definition. For $1 \leq p<\infty$, let $\sigma_{p}(\alpha)$ be the supremum of the numbers $\sigma$ for which there is a constant $C$ with

$$
\left(\int_{\mathbb{S}^{1}}|\widehat{\mu}(R \xi)|^{p} d \sigma(\xi)\right)^{1 / p} \leq C R^{-\sigma} \sqrt{I_{\alpha}(\mu)},
$$

for all $\mu$ supported in the unit ball $\mathbb{B} \subset \mathbb{R}^{2}$ and all $R>0$. Here $\mathbb{S}^{1}$ denotes the unit circle in $\mathbb{R}^{2}$. The problem of estimating $\sigma_{p}$ has been of interest in recent years largely because of its connection with certain longstanding conjectures in Geometric Measure Theory. See [13, 14, [9, and 8. The inequalities (11) have been studied explicitly by many authors, in particular, Bourgain, Mattila, Sjölin, and Wolff (see [4], 10], [11, and [13], respectively).

In addition, inequalities of the form (11) may be interpreted via duality as examples of weighted estimates for the extension operator (dual to the Fourier Restriction operator). Inequalities such as (1), and in particular the work of Wolff [13], have generated substantial interest from this perspective. See [5], 3], 6], and [2] for some related work.

Before we introduce our results we briefly discuss what is known about the exponents $\sigma_{p}(\alpha)$ for $0<\alpha \leq 2$ and $1 \leq p<\infty$.

Received by the editors April 27, 2001.

2000 Mathematics Subject Classification. Primary 42B10.

Key words and phrases. Fourier transforms, circular means, $\alpha$-energy.

(C)2002 American Mathematical Society 
For $p \geq 2, \sigma_{p}(\alpha)$ has been computed for each $0<\alpha \leq 2$. In particular

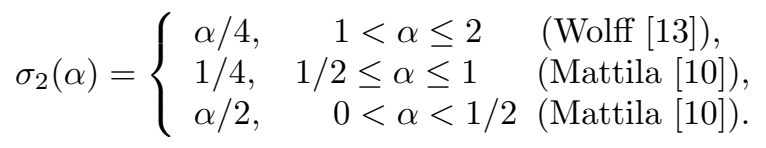

Wolff showed in [13] that for $p>2, \sigma_{p}$ is equal to what one might expect from interpolating between the $p=2$ and the trivial $p=\infty$ results.

For $1 \leq p<2$ the picture is incomplete. By the Cauchy-Schwarz inequality,

$$
\sigma_{p} \geq \sigma_{q} \text { for all } p \leq q,
$$

and hence

$$
\sigma_{p} \geq \sigma_{2} \text { for all } 1 \leq p<2 .
$$

As yet, this 'trivial' bound for $\sigma_{p}(1 \leq p<2)$ is the only lower bound that is known.

For $p=1$, the upper bound

$$
\sigma_{1}(\alpha) \leq \min \left\{\frac{\alpha}{2}, \frac{1}{3}+\frac{\alpha}{12}\right\}
$$

was obtained by Wolff in [13] using a certain randomisation argument.

In this article we focus on the case $p=1$, i.e. the problem of estimating the exponent $\sigma_{1}$. Our approach (motivated by that of Wolff [13]) involves the introduction of a broad family of randomised versions of this problem which contains the original problem as an 'end point'. For certain members of this family we are able to find the corresponding sharp exponent. These results provide a new upper bound for $\sigma_{1}$ as a corollary.

In addition, we give an alternative proof of this corollary which we believe to be enlightening since it involves 'less randomising' and provides an interesting interpretation of the geometry contained in the proof of our main result.

We now turn to the details, beginning with a sequence of reductions.

By definition, $\sigma_{1}(\alpha)$ is the supremum of the numbers $\sigma$ for which there is a constant $C$ with

$$
\int_{\mathbb{S}^{1}}|\widehat{\mu}(R \xi)| d \sigma(\xi) \leq C R^{-\sigma} \sqrt{I_{\alpha}(\mu)},
$$

for all measures $\mu$ supported in the unit ball $\mathbb{B}$ and $R>0$. By duality, (3) is equivalent to

$$
\int_{\mathbb{R}^{2}}|\widehat{f d \sigma}(R \xi)| d \mu(\xi) \leq C R^{-\sigma} \sqrt{I_{\alpha}(\mu)}\|f\|_{\infty},
$$

for all $f \in L^{\infty}\left(\mathbb{S}^{1}\right), R>0$, and $\operatorname{supp}(\mu) \subset \mathbb{B}$. Furthermore, (4) can be seen to be equivalent to

$$
\int_{\mathbb{R}^{2}}|\widehat{\phi}| d \mu \leq C R^{-\sigma+1} \sqrt{I_{\alpha}(\mu)}\|\phi\|_{\infty},
$$

for all $\phi \in L^{\infty}\left(\mathbb{R}^{2}\right)$ with $\operatorname{supp}(\phi) \subset\left\{x \in \mathbb{R}^{2}:|| x|-R| \leq 1\right\}$. The equivalence of (3), (4) and (15) is clarified in [13].

Let $0 \leq \gamma \leq 1 / 2$. We now decompose the annulus

$$
A=\left\{x \in \mathbb{R}^{2}:|| x|-R| \leq 1\right\}
$$


into a disjoint union

$$
A=\bigcup_{k=1}^{\left[R^{\gamma}\right]} A_{k}
$$

where $A_{k}$ is the segment of annulus

$$
\left\{x \in A: 0<2 \pi k\left[R^{\gamma}\right]^{-1}-\arg x \leq 2 \pi\left[R^{\gamma}\right]^{-1}\right\},
$$

and we write

$$
\phi=\sum_{k=1}^{\left[R^{\gamma}\right]} \phi_{k}, \text { where } \phi_{k}=\phi \chi_{A_{k}} .
$$

Kintchine's inequality applied to (5) implies

$$
\int_{\mathbb{R}^{2}}\left(\sum_{j=1}^{\left[R^{\gamma}\right]}\left|\widehat{\phi_{k}}\right|^{2}\right)^{1 / 2} d \mu \leq C R^{-\sigma+1} \sqrt{I_{\alpha}(\mu)}\|\phi\|_{L^{\infty}\left(\mathbb{R}^{2}\right)},
$$

for all $\sigma<\sigma_{1}(\alpha)$. For $0 \leq \gamma \leq 1 / 2$ and $0<\alpha \leq 2$, we define $\sigma^{\gamma}(\alpha)$ to be the supremum of the numbers $\sigma$ for which there is a constant $C$ such that (6) holds for all $\phi \in L^{\infty}(A), R>0$, and $\operatorname{supp} \mu \subset \mathbb{B}$. (The case $\gamma=0$ of (6) is equal to inequality (5), i.e. $\sigma^{0}=\sigma_{1}$.)

Notation. Throughout this article we use the letters $c$ and $C$ to denote constants that may change from line to line. However, we allow these constants to depend on the parameters $\alpha$ and $\gamma$.

Theorem 1. For $1 \leq \alpha \leq 2$ and $\frac{\alpha}{\alpha+2} \leq \gamma \leq \frac{1}{2}$,

$$
\sigma^{\gamma}(\alpha)=\frac{\alpha}{4}+\frac{\gamma(2-\alpha)}{4} .
$$

Corollary 2. For $1 \leq \alpha \leq 2$,

$$
\sigma_{1}(\alpha) \leq \frac{\alpha}{\alpha+2} .
$$

In [13] Wolff used (6) with $\gamma=1 / 2$ to prove the upper bound for $\sigma_{1}$ given by (2). In order to do this Wolff reduced (6) (for $\gamma=1 / 2$ ) further by using the observation that given any collection of rectangles $\left\{T_{k}\right\}$ of dimensions $1 \times R^{-1 / 2}$, such that the long side of $T_{k}$ has direction $e^{2 \pi i k R^{-1 / 2}}$, one can choose $\phi$, with $\|\phi\|_{\infty} \leq 1$, so that

$$
\left|\widehat{\phi}_{k}\right| \geq c R^{1 / 2} \chi_{T_{k}} .
$$

In this case (6) implies that

$$
\int_{\mathbb{R}^{2}}\left(\sum_{k} \chi_{T_{k}}\right)^{1 / 2} d \mu \leq C R^{-\sigma+1 / 2} \sqrt{I_{\alpha}(\mu)},
$$

for all such families of rectangles $\left\{T_{k}\right\}$. A particular choice of rectangles $\left\{T_{k}\right\}$ and measure $\mu$ now leads to Wolff's result. The case $\gamma=1 / 2$ is in a sense at a natural scale since the corresponding segments of annulus $\left\{A_{j}\right\}$ are in fact 'rectangles'. Theorem 1 confirms that the randomised inequality (6) at the 'natural scale' $\gamma=$ $1 / 2$ cannot provide extremal examples for inequality (5) (and thus inequality (3)).

In order to prove Corollary 2 from Theorem 10 one only needs to use the fact that $\sigma^{\gamma}(\alpha) \leq \frac{\alpha}{4}+\frac{\gamma(2-\alpha)}{4}$. In the proof of Theorem 1 , the example that we use to 
obtain this inequality involves an argument using rectangles similar to that of the previous paragraph. The alternative proof of Corollary 2] that we give at the end, is interesting as it avoids any direct consideration of rectangles.

The following lemma of Wolff [13] will be important to us.

Lemma 3. If $\mu$ is a measure supported in the unit ball in $\mathbb{R}^{2}$ and $0<\alpha \leq 2$, then we can decompose $\mu$ as a sum of $O(\log R)$ measures $\mu_{j}$, so that for each $j$,

$$
\mu_{j}\left(\mathbb{R}^{2}\right) \sup _{x \in \mathbb{R}^{2}} \sup _{r>R^{-1}}\left(\frac{\mu_{j}(B(x ; r))}{r^{\alpha}}\right) \leq C I_{\alpha}(\mu) .
$$

\section{The Proof of Theorem 1}

In order to prove the theorem, we will show that (6) holds for all $\sigma<\frac{\alpha}{4}+\gamma \frac{2-\alpha}{4}$, with $0<\alpha<2$ and $0<\gamma \leq 1 / 2$, and give an example where (6) fails for any $\sigma>\frac{\alpha}{4}+\gamma \frac{2-\alpha}{4}$, with $\gamma \geq \alpha /(2+\alpha)$ and $1 \leq \alpha \leq 2$.

We begin with the counterexample. Let $F=\left\{T_{k}\right\}_{k=1}^{\left[R^{\gamma}\right]}$ be a collection of rectangles of dimensions $R^{2 \gamma-1} \times R^{\gamma-1}$, so that the direction of the longest side of $T_{k}$ is given by $e^{2 \pi i k\left[R^{\gamma}\right]^{-1}}$. Then we take (as we may) $\phi \in L^{\infty}$, with $\|\phi\|_{L^{\infty}} \leq 1$, such that $\left|\widehat{\phi_{k}}(\xi)\right| \geq c \chi_{T_{k}}(\xi) R^{1-\gamma}$. Hence, (6) gives in this particular case (see also [13])

$$
\int\left(\sum \chi_{T_{k}}\right)^{1 / 2} d \mu \leq C R^{\gamma-\sigma} \sqrt{I_{\alpha}(\mu)}
$$

It suffices to show that (7) fails for $\sigma>\alpha / 4+\gamma(2-\alpha) / 4$ when $\gamma \geq \alpha /(2+\alpha)$ and $1 \leq \alpha \leq 2$.

Take $\delta=(1-\gamma)(\alpha-1)$. Consider the annulus

$$
B=\left\{x \in \mathbb{R}^{2}:|| x\left|-\frac{1}{10} R^{\delta}\right| \leq \frac{1}{10} R^{\gamma-1}\right\} .
$$

For $j=1, \ldots,\left[R^{\delta}\right]$ define

$$
B_{j}=\left\{x \in B: \arg (x) \in\left(2 \pi(j-1) R^{-\delta}, 2 \pi j R^{-\delta}\right)\right\}
$$

and its 'concentric half'

$$
B_{j}^{*}=\left\{x \in B: \arg (x) \in\left(2 \pi(j-3 / 4) R^{-\delta}, 2 \pi(j-1 / 4) R^{-\delta}\right)\right\} .
$$

We now translate $B_{j}^{*}$ to the unit ball, defining $\widetilde{B_{j}}=B_{j}^{*}-a_{j}$, where

$$
a_{j}=\left(\frac{1}{10} R^{\delta} \cos \left(2 \pi j R^{-\delta}\right), \frac{1}{10} R^{\delta} \sin \left(2 \pi j R^{-\delta}\right)\right) .
$$

Set $\widetilde{B}=\bigcup \widetilde{B_{j}}$ and $d \mu(x)=\chi_{\widetilde{B}}(x) d x .(\widetilde{B}$ looks like a 'curved fan'; see Figure 1.)

Next we observe that

$$
\begin{aligned}
I_{\alpha}(\mu) & =\int_{\mathbb{R}^{2}}\left(\int_{\mathbb{R}^{2}} \frac{d \mu(y)}{|x-y|^{\alpha}}\right) d \mu(x) \\
& \leq C \int_{\mathbb{R}^{2}}\left(\sum_{j=0}^{\infty} \frac{\mu\left(B\left(x ; 2^{-j}\right)\right)}{2^{-j \alpha}}\right) d \mu(x) \\
& \leq C \mu(\mathbb{B}) \sup _{x \in \mathbb{B}}\left(\sum_{j=0}^{\infty} \frac{\mu\left(B\left(x ; 2^{-j}\right)\right)}{2^{-j \alpha}}\right) .
\end{aligned}
$$




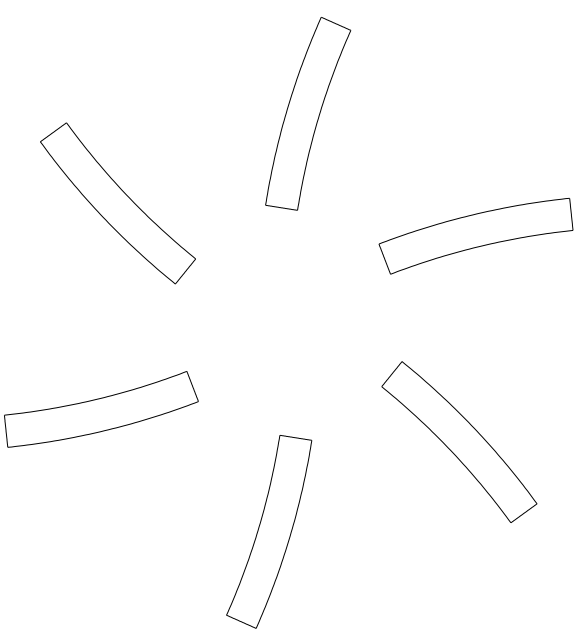

Figure 1. The 'curved fan'.

One can easily check that, for this choice of $\delta$,

$$
\mu(\mathbb{B}) \approx R^{(2-\alpha)(\gamma-1)} \approx \sup _{x \in \mathbb{B}}\left(\sum_{j=0}^{\infty} \frac{\mu\left(B\left(x ; 2^{-j}\right)\right)}{2^{-j \alpha}}\right),
$$

and hence, $\left[I_{\alpha}(\mu)\right]^{1 / 2} \approx R^{(2-\alpha)(\gamma-1)}$. (As usual, for $X, Y \geq 0$, we say that $X \approx Y$ if there exists a constant $C>0$ such that $C^{-1} Y \leq X \leq C Y$. Of course, this constant $C$ may depend on the parameters $\alpha$ and $\gamma$.)

We choose the rectangle $T_{k}$ to be tangent to $\widetilde{B}$ in the following way. For each $k$, we define the point

$$
b_{k}=\left(-\sin \left(2 \pi k\left[R^{\gamma}\right]^{-1}\right), \cos \left(2 \pi k\left[R^{\gamma}\right]^{-1}\right)\right) .
$$

Then, if $2 \pi k\left[R^{\gamma}\right]^{-1}+\pi / 2 \in\left[2 \pi(j-1) R^{-\delta}, 2 \pi j R^{-\delta}\right)$, we take $T_{k}$ to be centred at the point $-a_{j}+b_{k} R^{\delta} / 10$. Since $-\gamma \leq-\delta$, every $j$ corresponds to $C R^{\gamma-\delta}$ rectangles satisfying

$$
\left|T_{k} \cap \widetilde{B_{j}}\right| \approx R^{\gamma-1} R^{(-1+\gamma+\delta) / 2}
$$

Moreover, if $\gamma \geq \alpha /(2+\alpha)$,

We then have

$$
\sum \chi_{T_{k}}(x) \approx R^{\gamma(2+\alpha) / 2-\alpha / 2} \quad \text { for all } x \in \widetilde{B} \text {. }
$$

$$
\int\left(\sum \chi_{T_{k}}\right)^{1 / 2} d \mu \approx R^{\gamma(2+\alpha) / 4-\alpha / 4} \mu(\mathbb{B}) \approx R^{\gamma(2+\alpha) / 4-\alpha / 4} \sqrt{I_{\alpha}(\mu)} .
$$

Therefore, for (7) to hold, $\sigma \leq \gamma-(\gamma(2+\alpha) / 4-\alpha / 4)=\gamma(2-\alpha) / 4+\alpha / 4$.

Now we show that (6) holds for all $\sigma<\frac{\alpha}{4}+\gamma \frac{2-\alpha}{4}$. The argument uses ideas of A. Córdoba [7] and T. Wolff [13]. Let us suppose, as we may, that $\|\phi\|_{\infty}=1$.

We begin by using the Cauchy-Schwarz inequality

$$
\int\left(\sum\left|\widehat{\phi_{k}}\right|^{2}\right)^{1 / 2} d \mu \leq\left(\int \sum\left|\widehat{\phi_{k}}\right|^{2} d \mu\right)^{1 / 2} \mu(\mathbb{B})^{1 / 2}
$$


Note that, since supp $\phi_{k} \subset A_{k}$ is contained in a ball of radius $c R^{1-\gamma}$, then

$$
\int\left|\widehat{\phi_{k}}\right|^{2} d \mu=\int\left|\widehat{\phi_{k}}\right|^{2} d \mu_{R^{\gamma-1}}
$$

where $\mu_{R^{\gamma-1}}=R^{2(1-\gamma)} \Psi\left(R^{(1-\gamma)}\right.$. $) * \mu$ for some $\Psi \in \mathcal{S}\left(\mathbb{R}^{2}\right)$. Hence,

$$
\int\left(\sum\left|\widehat{\phi_{k}}\right|^{2}\right)^{1 / 2} d \mu \leq\left(\int \sum\left|\widehat{\phi_{k}}\right|^{2} d \mu_{R^{\gamma-1}}\right)^{1 / 2} \mu(\mathbb{B})^{1 / 2} .
$$

By Cauchy-Schwarz and Hölder's inequality,

$$
\begin{aligned}
\left(\int \sum\left|\widehat{\phi_{k}}\right|^{2} d \mu_{R^{\gamma-1}}\right)^{1 / 2} \mu(\mathbb{B})^{1 / 2} & \leq\left(\int\left(\sum\left|\widehat{\phi_{k}}\right|^{2}\right)^{2}\right)^{1 / 4}\left\|\mu_{R^{\gamma-1}}\right\|_{L^{2}\left(\mathbb{R}^{2}\right)}^{1 / 2} \mu(\mathbb{B})^{1 / 2} \\
\leq & \left(\int\left(\sum\left|\widehat{\phi_{k}}\right|^{2}\right)^{2}\right)^{1 / 4}\left\|\mu_{R^{\gamma-1}}\right\|_{L^{\infty}\left(\mathbb{R}^{2}\right)}^{1 / 4}\left\|\mu_{R^{\gamma-1}}\right\|_{L^{1}\left(\mathbb{R}^{2}\right)}^{1 / 4} \mu(\mathbb{B})^{1 / 2} \\
& \leq\left(\int\left(\sum\left|\widehat{\phi_{k}}\right|^{2}\right)^{2}\right)^{1 / 4}\left\|\mu_{R^{\gamma-1}}\right\|_{L^{\infty}\left(\mathbb{R}^{2}\right)}^{1 / 4} \mu(\mathbb{B})^{3 / 4} \\
& \leq C\left(\int\left(\sum\left|\widehat{\phi_{k}}\right|^{2}\right)^{2}\right)^{1 / 4}\left[R^{(2-\alpha)(1-\gamma)} \sup _{r \geq R^{\gamma-1}} \frac{\mu\left(B_{r}\right)}{r^{\alpha}}\right]^{1 / 4} \mu(\mathbb{B})^{3 / 4},
\end{aligned}
$$

where the supremum is taken over all balls of radius $r \geq R^{\gamma-1}$ contained in the unit ball. The last inequality above is a simple consequence of the rapid decay of $|\Psi|$.

Take $1 \leq m \leq 2$ such that $m\left[R^{\gamma}\right]^{-1} / R^{-1 / 2} \in \mathbb{N}$. We now consider a further subdivision of the annulus $A$,

$$
A=\bigcup_{l=1}^{m R^{1 / 2}} \tilde{A}_{l},
$$

where $\tilde{A}_{l}$ is the segment of annulus

$$
\left\{x \in A: 0 \leq 2 \pi l R^{-1 / 2} / m-\arg x<2 \pi R^{-1 / 2} / m\right\},
$$

and we write

$$
\phi=\sum_{l=1}^{m R^{1 / 2}} \Phi_{l}, \text { where } \Phi_{l}=\phi \chi_{\tilde{A}_{l}} .
$$

Then, a well known orthogonality argument originally due to Córdoba (see [7] and 13] Lemma 1.3) shows that

$$
\int\left(\sum_{k}\left|\widehat{\phi_{k}}\right|^{2}\right)^{2} \leq C \int\left(\sum_{l}\left|\widehat{\Phi_{l}}\right|^{2}\right)^{2}
$$

Now, by Plancherel's Theorem,

$$
\int\left(\sum\left|\widehat{\Phi_{l}}\right|^{2}\right)^{2}=\int \sum_{l} \sum_{j}\left|\widehat{\Phi_{l}}\right|^{2}\left|\widehat{\Phi_{j}}\right|^{2}=\sum_{l, j} \int\left|\Phi_{l} * \widetilde{\Phi_{j}}\right|^{2} .
$$


By the choice of $\left\{\tilde{A}_{l}\right\}_{l=1}^{m R^{1 / 2}}$,

$$
\left\|\Phi_{l} * \widetilde{\Phi_{j}}\right\|_{L^{\infty}\left(\mathbb{R}^{2}\right)} \leq\|\phi\|_{L^{\infty}\left(\mathbb{R}^{2}\right)}^{2} \sup _{a \in \mathbb{R}^{2}}\left|\tilde{A}_{l} \cap\left(a+\tilde{A}_{j}\right)\right| \leq C\|\phi\|_{L^{\infty}\left(\mathbb{R}^{2}\right)}^{2} \frac{R^{1 / 2}}{|j-l|+1} .
$$

Moreover,

$$
\left|\operatorname{supp}\left(\Phi_{l} * \widetilde{\Phi_{j}}\right)\right| \leq\left|\operatorname{supp} \Phi_{j}+\operatorname{supp} \widetilde{\Phi_{l}}\right| \leq C R^{1 / 2}[|j-l|+1], \quad \text { for all } l, j .
$$

Hence,

$$
\begin{aligned}
\sum_{l, j} \int\left|\Phi_{l} * \widetilde{\Phi_{j}}\right|^{2} & \leq C \sum_{l, j}\left(\frac{R^{1 / 2}}{|j-l|+1}\right)^{2} R^{1 / 2}[|j-l|+1] \\
& =C R^{3 / 2} \sum_{l, j=1}^{m R^{1 / 2}} \frac{1}{|j-l|+1} \\
& \leq C R^{2} \log R .
\end{aligned}
$$

From this, we conclude that

$$
\begin{aligned}
\int\left(\sum\left|\widehat{\phi_{k}}\right|^{2}\right. & 1 / 2 \\
& \leq C R^{1 / 2}(\log R)^{1 / 4} R^{(2-\alpha)(1-\gamma) / 4} \sup _{r \geq R^{\gamma-1}}\left[\frac{\mu\left(B_{r}\right)}{r^{\alpha}}\right]^{1 / 4} \mu(\mathbb{B})^{3 / 4},
\end{aligned}
$$

and so, by Lemma 3

$$
\int\left(\sum\left|\widehat{\phi_{k}}\right|^{2}\right)^{1 / 2} d \mu \leq C R^{-\gamma(2-\alpha) / 4-\alpha / 4+1}(\log R)^{5 / 4} \sqrt{I_{\alpha}(\mu)} .
$$

Observe that this has incurred an additional factor of $\log R$. Hence, (6) holds for any $\sigma<\frac{\alpha}{4}+\gamma \frac{2-\alpha}{4}$, and therefore, $\sigma^{\gamma}(\alpha) \geq \frac{\alpha}{4}+\gamma \frac{2-\alpha}{4}$.

\section{An alternative proof of Corollary 2}

For us, the Bessel function $J_{s}$ will be given by

$$
J_{s}(t)=\int_{0}^{2 \pi} e^{i(s \theta-t \sin \theta)} d \theta
$$

for each $t \in \mathbb{R}$. For this we have the following estimate.

Lemma 4. There exists an absolute constant $c>0$ such that whenever $s \geq 1$ and $|s-t| \leq c s^{1 / 3}$,

$$
J_{s}(t) \geq C s^{-1 / 3} \text {. }
$$

Proof. The proof of Lemma 4 can be seen as a consequence of the Mean Value Theorem applied to the following two estimates (see [12] page 260, and [1, respectively):

(i) $J_{s}(s) \geq C s^{-1 / 3}$ for all $s \geq 1$, and

(ii) $\left|J_{s}^{\prime}(t)\right| \leq C s^{-2 / 3}$ for $|s-t| \leq s^{1 / 3}$. 
We begin by considering the relatively simple case of $\alpha=1$. Let $\sigma<\sigma_{1}(1)$. By definition,

$$
\int_{\mathbb{R}^{2}}|\widehat{g d \sigma}(R \xi)| d \mu \leq C R^{-\sigma}\|g\|_{\infty} \sqrt{I_{1}(\mu)},
$$

for all $R>0, g \in L^{\infty}\left(\mathbb{S}^{1}\right)$, and measures $\mu$ supported in the unit ball.

We now observe that if $g(x)=e^{i R \arg x}$, and $d \mu(\xi)=\chi_{A}(\xi) d \xi$, where

$$
A=\left\{\xi: 0 \leq 1-|\xi| \leq c R^{-2 / 3}\right\},
$$

then by Lemma 4

$$
|\widehat{g d \sigma}(R \xi)|=\left|J_{R}(R|\xi|)\right| \geq C R^{-1 / 3}
$$

on $A$. Proceeding as in (8) we may also show that $I_{1}(\mu) \leq C(\log R) \mu(\mathbb{B})^{2}$. Now, by (9) we conclude that $\sigma \leq 1 / 3$, and hence $\sigma_{1}(1) \leq 1 / 3$, as claimed.

Let $1<\alpha<2$ be fixed, and let $1<\beta<3 / 2$ be some parameter to be determined. (The exponent $\sigma_{1}(\alpha)$ is known for $\alpha=2$, as one may observe in the Introduction.) Let $\sigma<\sigma_{1}(\alpha)$. We now define $g \in L^{\infty}\left(\mathbb{S}^{1}\right)$ by

$$
g(x)=\sum_{j} \chi_{\tau_{j}}(\arg x-\pi / 2) e^{i\left(R^{\beta} \arg x+R x \cdot \xi_{j}\right)},
$$

where

$$
\tau_{j}=\left[2 \pi(j-1) R^{1-\beta}, 2 \pi j R^{1-\beta}\right),
$$

and $\xi_{j} \in \mathbb{R}^{2}$ is given by

$$
\xi_{j}=R^{\beta-1}\left(\cos 2 \pi j R^{1-\beta}, \sin 2 \pi j R^{1-\beta}\right),
$$

for $j=1, \ldots,\left[R^{\beta-1}\right]$.

Now,

$$
\begin{aligned}
\widehat{g d \sigma}(R \xi) & =\sum_{j} \int_{0}^{2 \pi} \chi_{\tau_{j}}(\theta-\pi / 2) e^{i\left(R^{\beta} \theta+R\left(\xi-\xi_{j}\right) \cdot(\cos \theta, \sin \theta)\right)} d \theta \\
& =\sum_{j} e^{i R^{\beta}\left(\arg \left(\xi-\xi_{j}\right)-\pi / 2\right)} \int_{0}^{2 \pi} \chi_{\tau_{j}}\left(\theta+\arg \left(\xi-\xi_{j}\right)\right) e^{i\left(R^{\beta} \theta-R\left|\xi-\xi_{j}\right| \sin \theta\right)} d \theta .
\end{aligned}
$$

Using (4) and Kintchine's Inequality we obtain

$$
\begin{aligned}
& \int_{\mathbb{R}^{2}}\left(\sum_{j}\left|\int_{0}^{2 \pi} \chi_{\tau_{j}}\left(\theta+\arg \left(\xi-\xi_{j}\right)\right) e^{i\left(R^{\beta} \theta-R\left|\xi-\xi_{j}\right| \sin \theta\right)} d \theta\right|^{2}\right)^{1 / 2} d \mu(\xi) \\
& \quad \leq C R^{-\sigma} \sqrt{I_{\alpha}(\mu)},
\end{aligned}
$$

for all $R>0$ and measures $\mu$ supported in the unit ball. Notice that after rescaling, this is equivalent to applying (6) with $\gamma=1-\beta$.

\section{Lemma 5.}

$$
\left|\int_{0}^{2 \pi} \chi_{\tau_{j}}(\theta+\phi) e^{i\left(R^{\beta} \theta-R t \sin \theta\right)} d \theta\right| \geq C R^{-\beta / 3}
$$

on

$$
\left\{(t, \phi):\left|t-R^{\beta-1}\right| \leq c R^{\beta / 3-1} \text { and } \phi \in \tau_{j}^{*}\right\},
$$


where

$$
\tau_{j}^{*}=\left[2 \pi(j-3 / 4) R^{1-\beta}, 2 \pi(j-1 / 4) R^{1-\beta}\right)
$$

denotes the 'concentric half' of $\tau_{j}$.

Before we prove Lemma 5 we show how it can be used to complete the proof of the theorem.

By Lemma 5

$$
\left|\int_{0}^{2 \pi} \chi_{\tau_{j}}\left(\theta+\arg \left(\xi-\xi_{j}\right)\right) e^{i\left(R^{\beta} \theta-R\left|\xi-\xi_{j}\right| \sin \theta\right)} d \theta\right| \geq C R^{-\beta / 3}
$$

on the segment of annulus

$$
F_{j}:=\left\{\xi \in \mathbb{R}^{2}:|| \xi-\xi_{j}\left|-R^{\beta-1}\right| \leq c R^{\beta / 3-1} ; \arg \left(\xi-\xi_{j}\right) \in \tau_{j}^{*}\right\},
$$

and hence

$$
\left(\sum_{j}\left|\int_{0}^{2 \pi} \chi_{\tau_{j}}\left(\theta+\arg \left(\xi-\xi_{j}\right)\right) e^{i\left(R^{\beta} \theta-R\left|\xi-\xi_{j}\right| \sin \theta\right)} d \theta\right|^{2}\right)^{1 / 2} \geq C R^{-\beta / 3}
$$

on the 'curved fan' (see Figure 1)

$$
F=\bigcup_{j} F_{j}
$$

Now, if we take $d \mu(x)=\chi_{F}(x) d x$, then (10) implies that

$$
R^{-\beta / 3}|F| \leq C R^{-\sigma} \sqrt{I_{\alpha}(\mu)}
$$

A simple geometrical argument similar to that in the proof of Theorem 1 shows that

$$
I_{\alpha}(\mu) \approx|F|\left(|F|+\sup _{r=R^{\beta / 3-1}} \frac{\mu\left(B_{r}\right)}{r^{\alpha}}\right) .
$$

Hence $\sigma \leq \beta / 3$, provided $\beta$ is chosen so that

$$
\sup _{r=R^{\beta / 3-1}}\left(\frac{\mu\left(B_{r}\right)}{r^{\alpha}}\right) \leq C|F|,
$$

which is the case provided $\beta$ satisfies

$$
\left(R^{\beta / 3-1}\right)^{2-\alpha} \leq C R^{\beta-1} R^{\beta / 3-1},
$$

which occurs whenever

$$
\beta \geq \frac{3 \alpha}{\alpha+2}
$$

We may therefore conclude that

$$
\sigma \leq \frac{\alpha}{\alpha+2}
$$

and hence

$$
\sigma_{1}(\alpha) \leq \frac{\alpha}{\alpha+2},
$$

for $1<\alpha<2$.

Remark. The above choice of $\beta$ is optimal subject to our constraints. 
We now turn to the proof of Lemma 5. Suppose $\left|t-R^{\beta-1}\right| \leq c R^{\beta / 3-1}$ and $\phi \in \tau_{j}^{*}$. Now,

$$
\begin{aligned}
\left|\int_{0}^{2 \pi} \chi_{\tau_{j}}(\theta+\phi) e^{i\left(R^{\beta} \theta-R t \sin \theta\right)} d \theta\right| \\
\geq\left|J_{R^{\beta}}(R t)\right|-\left|\int_{0}^{2 \pi}\left[1-\chi_{\tau_{j}}(\theta+\phi)\right] e^{i\left(R^{\beta} \theta-R t \sin \theta\right)} d \theta\right| .
\end{aligned}
$$

Hence, by Lemma 4, it suffices to show that

$$
\left|\int_{0}^{2 \pi}\left[1-\chi_{\tau_{j}}(\theta+\phi)\right] e^{i\left(R^{\beta} \theta-R t \sin \theta\right)} d \theta\right|<<R^{-\beta / 3},
$$

for all $\left|t-R^{\beta-1}\right| \leq c R^{\beta / 3-1}$ and $\phi \in \tau_{j}^{*}$.

To this end let $h(\theta)=R^{\beta} \theta-R t \sin \theta$. Now, $\theta+\phi \in \tau_{j}^{c}$ and $\phi \in \tau_{j}^{*}$ imply that $|\theta| \geq c R^{1-\beta}$. A simple calculation shows that for $\operatorname{such} \theta,\left|h^{\prime}(\theta)\right| \geq c R^{2-\beta}$. Hence, integration by parts gives

$$
\left|\int_{0}^{2 \pi}\left[1-\chi_{\tau_{j}}(\theta+\phi)\right] e^{i\left(R^{\beta} \theta-R t \sin \theta\right)} d \theta\right| \leq c R^{\beta-2},
$$

for all $\left|t-R^{\beta-1}\right| \leq c R^{\beta / 3-1}$ and $\phi \in \tau_{j}^{*}$. Since $\beta<3 / 2, R^{\beta-2}<<R^{-\beta / 3}$ for large $R$, as required.

\section{ACKNOWLEDGEMENTS}

The first author was supported by the European Commission via the TMR network (Harmonic Analysis). The second author was partially supported by the Spanish DGICYT (grant number PB97-0030) and the European Commission via the TMR network (Harmonic Analysis).

\section{REFERENCES}

1. J. A. Barceló, Tesis Doctoral, Universidad Autónoma de Madrid, 1988.

2. J. A. Barceló, J. M. Bennett, A. Carbery, A bilinear extension estimate in two dimensions, preprint (2001).

3. J. A. Barceló, A. Ruiz, L. Vega, Weighted estimates for the Helmholtz equation and consequences, JFA Vol. 150 (1997), 2, 356-382. MR 99a:35033

4. J. Bourgain, Hausdorff dimension and distance sets, Israel J. Math. 87 (1994), 193-201. MR 95h:28008

5. A. Carbery, F. Soria, Pointwise Fourier inversion and localisation in $\mathbb{R}^{n}$, Journal of Fourier Analysis and Applications 3, special issue, 847-858 (1997). MR 99c:42018

6. A. Carbery, F. Soria, A. Vargas, preprint.

7. A. Córdoba, The Kakeya Maximal Functions and the Spherical Summation Multipliers, Am. J. Math. 99 (1977), 1-22. MR 56:6259

8. N. H. Katz, T. Tao, Some connections between Falconer's distance set conjecture, and sets of Furstenburg type, New York J. Math. 7 (2001), 149-187.

9. P. Mattila, Geometry of Sets and Measures in Euclidean Spaces, Cambridge Studies in Advanced Mathematics 44. MR 96h:28006

10. P. Mattila, Spherical averages of Fourier transforms of measures with finite energy; dimensions of intersections and distance sets, Mathematika 34 (1987), 207-228. MR 90a:42009

11. P. Sjölin, Estimates of spherical averages of Fourier transforms and dimensions of sets, Mathematika 40 (1993), 322-330. MR 95f:28007

12. G. N. Watson, A Treatise on the Theory of Bessel Functions, Cambridge University Press, 1944. MR 6:64a 
13. T. H. Wolff, Decay of circular means of Fourier transforms of measures, Internat. Math. Res. Notices 10 (1999), 547-567. MR 2000k:42016

14. T. H. Wolff, Recent work connected with the Kakeya problem, Prospects in mathematics (Princeton, NJ, 1996), 129-162, Amer. Math. Soc., Providence, RI, 1999. MR 2000d:42010

Department of Mathematics, University Autonoma de Madrid, 28049 Madrid, Spain

E-mail address: jonathan.bennett@uam.es

Department of Mathematics, University Autonoma de Madrid, 28049 Madrid, Spain

E-mail address: ana.vargas@uam.es 